\title{
SYMMETRY AND MONOTONICITY PROPERTIES \\ FOR POSITIVE SOLUTIONS OF SEMI-LINEAR ELLIPTIC PDE'S.
}

\author{
by \\ Jean Dolbeault \\ Ceremade UMR CNRS No 7534 \\ Université Paris IX-Dauphine \\ Place du Maréchal de Lattre de Tassigny \\ 75775 Paris Cédex 16, FRANCE \\ and \\ Patricio Felmer ${ }^{1}$ \\ Departamento de Ingeniería Matemática F.C.F.M. \\ Universidad de Chile \\ Casilla 170 Correo 3, Santiago, CHILE.
}

\footnotetext{
${ }^{1}$ Partially supported by ECOS-Conicyt, Cátedra Presidencial and FONDAP de Matemáticas Aplicadas
} 


\section{Introduction}

This article is devoted to the study of some qualitative properties of positive solutions to semilinear elliptic partial differential equations induced by the shape of the domain and the nature of the differential operator. For the boundary value problem

$$
\begin{cases}\Delta u+f(u)=0 & \text { in } \Omega \subset \mathbb{R}^{N}, \\ u>0 & \text { in } \Omega, \\ u=0 & \text { on } \partial \Omega,\end{cases}
$$

one expects that if $\Omega$ is a ball then $u$ is radially symmetric and decreasing along any radius, and if $\Omega$ is a convex cone then $u$ is increasing in the direction of the rays of the cone.

These qualitative properties have been studied by many authors starting with the fundamental work of Alexandroff [1], who introduced the moving planes method. This method was further developed by Serrin [18] and Gidas, $\mathrm{Ni}$ and Nirenberg [10] and [11]. Later, several other authors have devoted attention to these questions, without being exhaustive we mention the papers by Li [14], Berestycki and Nirenberg [2], Li and Ni [15], Esteban and Lions [9], and recent works by Berestycki, Caffarelli and Nirenberg, see [3] and references.

All the above mentioned works require the nonlinearity $f$ to be Lipschitz continuous, because of the use of the Maximum Principle. However this property can be relaxed, as suggested by some recent works. The earliest work we know in this direction is the one by Lions [16] where $f$ was assumed 
merely measurable, but non-negative and $N=2$. For nonlinearities changing sign we have a first work by Kaper, Kwong and Li [13]. Later appeared the papers by Gui [12] and Cortázar, Elgueta and Felmer [6] and [7]. In these works the Lipschitz property is lost at the origin, that is, $f$ is assumed to be locally Lipschitz in $(0,+\infty)$ and continuous in $[0,+\infty)$. The typical form of $f$ corresponds to the case $f(u)=-u^{q}+u^{p}$ with $0<q<1 \leq p$.

In a more recent work [8], we considered more general nonlinearities that in the simplest form correspond to functions $f$ satisfying the following hypothesis:

(f1) For any $s \in[0,+\infty[$, there exists a constant $\eta>0$ such that on $] s-$ $\eta, s+\eta\left[\cap \mathbb{R}^{+}, f\right.$ is either strictly decreasing or Lipschitz, and

(f2) If $f(s)=0$, then $f$ is locally Lipschitz in a neighborhood of $s$ in $\mathbb{R}^{+}$.

We proved that for $f$ satisfying (f1) and (f2) and for $\Omega=B(0,1)$, the unit ball, positive solutions of (1.1) are radial. Other related results were also obtained.

During the research leading to [8] we learned of a work of Brock [4] and [5], in which he has developed a continuous Steiner symmetrization procedure that defines a homotopy between the function and its Steiner symmetrization. Brock proves continuity properties for this operation in several spaces and also some inequalities for Dirichlet type integrals. When these properties are applied to (1.1) in case $\Omega$ is a ball and $f$ is continuous, a result on local symmetry properties of $u$ is obtained. It is shown that all solutions of (1.1) are locally symmetric. See Theorem 13 and Corollary 5 in [5]. This general structure theorem gives rise to some very interesting symmetry results when $f$ satisfies further hypotheses. See [5] for precise statements.

In view of the results in [5], and those using moving planes methods, an interesting question arises: is it possible to give a moving planes argument to obtain symmetry when the nonlinearity is merely continuous? This direct approach has several advantages: it applies directly to the study of 
global monotonicity questions in unbounded domains, it can be used to obtain monotonicity properties near the boundary of convex non-symmetric domains and it can be extended to some equations not having divergence form.

In this article we answer this question positively in dimension $N=2$ and we conjecture that the answer is positive regardless of the dimension.

Being more precise we assume that the nonlinearity is a function $f$ : $[0,+\infty) \rightarrow \mathbb{R}$ that satisfies:

(H1) The function $f$ is continuous in $[0,+\infty)$.

(H2) $f(0)>0$ or there exists a constant $c \in \mathbb{R}$ such that

$$
\liminf _{u>0, u \rightarrow 0} \frac{f(u)}{u} \geq c .
$$

(H3) If $\bar{u} \in] 0,+\infty)$ is such that $f(\bar{u})=0$, then there exists a constant $C \in \mathbb{R}$ such that

$$
\liminf _{u \neq v} \frac{f(u)-f(v)}{u-v} \geq C .
$$

For simplicity, we state our first theorem for strictly convex domains of class $C^{2}$. We refer to Theorem 3.1 for the general statement,

Theorem 1.1 Assume $\Omega \subset \mathbb{R}^{2}$ is a $C^{2}$ domain, symmetric with respect to the coordinate axis and with $\bar{\Omega}$ strictly convex. If $u \in C^{2}(\Omega) \cap C^{1}(\bar{\Omega})$ is a solution of (1.1) then $u$ is symmetric with respect to the coordinate axis and $\frac{\partial u}{\partial x_{i}}(x)<0$ for all $\left(x_{1}, x_{2}\right) \in \Omega$ with $x_{i}>0, i=1,2$.

Moreover, $\nabla u(x)=0$ if and only if $x=0$ and $\nabla u(x) \cdot \gamma<0$ for any $\gamma \in S^{1}$ and $x \in \Omega$ such that

$$
x \cdot \gamma>\sup \{y \cdot \gamma \mid y \in \partial \Omega, \quad \nu(y) \cdot \gamma=0\},
$$

where $\nu(y)$ is the outgoing normal at $y \in \partial \Omega$. 
Remark 1.1. It is not clear that this theorem can be obtained using the techniques introduced by Brock in [5], except if $\Omega$ is a ball. In such a case $u$ is radially symmetric and strictly decreasing.

Remark 1.2. As we already mentioned, the moving planes technique can be used to obtain monotonicity of positive solutions near the boundary for general domains. In fact, hypothesis (H2) implies this is true in a small neighborhood of the boundary. Theorem 1.1 and 3.1 say that this property is maintained up to reaching the geometric obstruction given by (1.2).

The proof of Theorem 1.1 is based on the moving planes (lines) technique. We propose a new definition of the moving plane condition (2.1), involving only the derivative of $u$ rather that its values. Under hypothesis (H2) we can start the argument in each direction. Then we analyze the situation when the plane reaches a critical position introducing a new way of using the Maximum Principle and the Hopf Lemma. Actually, taking advantage of a nonvanishing derivative and using a change of coordinates, $u$ plays the role of an independent variable, allowing the nonlinearity to be only continuous. Thus we show that the derivative of $u$ vanishes in the direction orthogonal to the plane. This idea was used by Peletier and Serrin [17] in the study of the uniqueness problem for radially symmetric solutions. Then we introduce a second idea, a rescaling argument to show that also the second derivative vanishes in that direction. Finally a perturbation analysis leads to show that $f(u)=0$ from where we can conclude using hypothesis (H3).

Remark 1.3 Theorem 1.1 can be extended to more general domains as we describe in Section 3. See also hypotheses (O1), (O2) in Section 2.

Our second result deals with monotonicity properties of $u$ in case $\Omega \subset \mathbb{R}^{2}$ is an unbounded domain. For simplicity let us assume that there is a smooth function $\varphi: \mathbb{R} \rightarrow \mathbb{R}$, satisfying that $\lim _{\left|x_{2}\right| \rightarrow \infty} \varphi\left(x_{2}\right)=-\infty$. Let us consider $\Omega$ defined as

$$
\Omega=\left\{\left(x_{1}, x_{2}\right) \in \mathbb{R}^{2} \mid x_{1}<\varphi\left(x_{2}\right)\right\} .
$$


Next we state our second main result. For a more general version see Theorem 3.2 in Section 3.

Theorem 1.2 Assume that $\Omega$ is defined as above and that $f$ satisfies (H1), (H2) and (H3). If $u \in C^{2}(\Omega) \cap C^{1}(\bar{\Omega})$ is a solution of (1.1) then $u$ is strictly decreasing in the $x_{1}$-direction, actually $\frac{\partial u}{\partial x_{1}}(x)<0$ for any $x \in \Omega$.

The proof of this theorem follows the lines of that of Theorem 1.1.

Remark 1.4 With minor modifications our method can be applied to other equations with the differential operator not in divergence form, actually fully nonlinear elliptic equations invariant under rotations. Without reaching the greatest generality we can mention for instance the case of the equation

$$
\begin{cases}\Delta u \pm|\nabla u|^{\alpha}+f(u)=0 & \text { in } \Omega \subset \mathbb{R}^{2} \\ u>0 & \text { in } \Omega, \\ u=0 & \text { on } \partial \Omega .\end{cases}
$$

with $\alpha \geq 0$. Theorems like 1.1 and 1.2 can be extended to this case assuming the same hypotheses on $\Omega$ and $f$.

\section{Preliminary Results.}

In this section we prove some preliminary lemmas that build up the proof of our results. This part of the analysis can be extended to higher dimensions, but for simplicity we prefer to keep it within the context of $\mathbb{R}^{2}$. We first recall the basic notation and we introduce the hypotheses on the domains.

Given $\lambda \in \mathbb{R}$ and $\gamma \in S^{1}$ we define the cap

$$
\Sigma_{\lambda}(\gamma)=\{x \in \Omega \mid \gamma \cdot x>\lambda\}
$$

the hyperplane (line)

$$
T_{\lambda}(\gamma)=\left\{x \in \mathbb{R}^{2} \mid \gamma \cdot x=\lambda\right\}
$$


and the reflection of $\Sigma_{\lambda}(\gamma)$ with respect to $T_{\lambda}(\gamma)$ as

$$
\tilde{\Sigma}_{\lambda}(\gamma)=\left\{x \in \mathbb{R}^{2} \mid 2(\lambda-\gamma \cdot x) \gamma+x \in \Sigma_{\lambda}(\gamma)\right\}
$$

Next we introduce convexity notions for the case of bounded domains, having in mind the hypotheses we need for our theorems. The case of unbounded domains will be treated in Section 3.

Given $\gamma \in S^{1}$, a set $\Omega$ is said to be $\gamma$-convex if for any $x \in \Omega$ the set $\{t \in \mathbb{R} \mid x+t \gamma \in \Omega\}$ is an interval. We observe that when $\Omega$ is $\gamma$-convex and symmetric with respect to $T_{\lambda_{s}}(\gamma), \lambda_{s} \in \mathbb{R}$, then $\tilde{\Sigma}_{\lambda}(\gamma) \subset \Omega$ for all $\lambda \geq \lambda_{s}$. Our more general version of Theorem 1.1 will be for domains $\Omega$ that satisfy the following assumptions

(O1) $\Omega$ satisfies the interior sphere condition and its boundary is of class $C^{1}$.

(O2) $\Omega$ is $\gamma$-convex and symmetric with respect to the line $T_{\lambda_{s}}(\gamma)$, and for all $\varepsilon>0$ there exists $\delta>0$ so that for any $d \in S^{1},|d-\gamma|<\delta$ we have $\tilde{\Sigma}_{\lambda}(d) \subset \Omega$ for all $\lambda>\lambda_{s}+\varepsilon$.

We may see (O1) as $\gamma$-convexity made locally robust.

For the rest of the section we will assume that $\Omega$ is bounded and satisfies hypothesis (O1) and (O2), and for simplicity we consider without loss of generality that $\gamma=e_{1}=(1,0)$, and the symmetry line of $\Omega$ is $T_{0}\left(e_{1}\right)$. We denote $\Sigma_{\lambda}=\Sigma_{\lambda}\left(e_{1}\right), T_{\lambda}\left(e_{1}\right)=T_{\lambda}$ and we assume that $\Sigma_{\lambda}=\emptyset$ if and only if $\lambda>1$. We also consider the reflection of $x \in \Omega$ with respect to $T_{\lambda}$ defined as $x_{\lambda}=\left(2 \lambda-x_{1}, x_{2}\right)$ and define $w_{\lambda}(x)=u_{\lambda}(x)-u(x)$ for $x \in \Sigma_{\lambda}$, where $u_{\lambda}(x)=u\left(x_{\lambda}\right)$.

Our first lemma is the starting step of our moving planes procedure.

Lemma 2.1 Consider a solution $u \in C^{2}(\Omega) \cap C^{1}(\bar{\Omega})$ of (1.1) and assume that $f$ satisfies (H1) and (H2). Then for any $\eta>0$ there exists $\varepsilon>0$ such that if $x=\left(x_{1}, x_{2}\right) \in \bar{\Omega}$ is such that $x_{1} \geq \eta$ and $\operatorname{dist}(x, \partial \Omega) \leq \varepsilon$, then $\frac{\partial u(x)}{\partial x_{1}}<0$. 
Proof: Thanks to hypothesis (H1), near the boundary of $\Omega$ and for $x_{1}>0$ we have either

$$
\Delta u \leq 0
$$

in case $f(0)>0$, or

$$
\Delta u+(c-1) u \leq \Delta u+\frac{f(u)}{u} u \leq 0,
$$

in case $f(0) \leq 0$. Let us assume that Lemma 2.1 is false. Then there exists $\eta>0$ and a sequence $\left\{x^{k}\right\}$ such that $x_{1}^{k}>\eta, x^{k} \rightarrow \partial \Omega$ and $\frac{\partial u}{\partial x_{1}}\left(x^{k}\right)=0$. Up to the extraction of a subsequence we have that $x^{k} \rightarrow \bar{x}=\left(\bar{x}_{1}, \bar{x}_{2}\right) \in \partial \Omega$ with $\bar{x}_{1} \geq \eta$ and $\frac{\partial u}{\partial x_{1}}(\bar{x})=0$. But, on the other hand if $\nu$ denotes the exterior normal to $\partial \Omega$ at $\bar{x}$ then we have $\frac{\partial u}{\partial \nu}(\bar{x})<0$ because of Hopf's Lemma and, since the tangent derivative is zero, we have $\nabla u(\bar{x})=\frac{\partial u}{\partial \nu}(\bar{x}) \nu$. Finally we see that from $(\mathrm{O} 2)$ the normal vector $\nu$ always has a positive component in the direction of $e_{1}$, thus $\frac{\partial u}{\partial x_{1}}(\bar{x})<0$.

Next we consider the critical moving plane by defining

$$
\bar{\lambda}=\inf \left\{\lambda \in(0,1) \mid \frac{\partial u}{\partial x_{1}}(\bar{x})<0 \quad \text { in } \quad \Sigma_{\lambda}\right\} .
$$

We observe that because of Lemma 2.1 we have that $\bar{\lambda}$ belongs to $[0,1)$.

In order to prove symmetry we have to show that $\bar{\lambda}=0$. Assume the contrary, that is $\bar{\lambda}>0$. Then there exists $\bar{x} \in T_{\bar{\lambda}}$ such that $\frac{\partial u}{\partial x_{1}}(\bar{x})=0$. Moreover, because of Lemma 2.1, for $\eta=\bar{\lambda}$ there exists $\varepsilon>0$ so that

$$
\bar{x} \in T_{\bar{\lambda}} \quad \text { and } \quad \frac{\partial u}{\partial x_{1}}(\bar{x})=0 \quad \text { implies } \operatorname{dist}(\bar{x}, \partial \Omega)>\varepsilon .
$$

In the next lemma we further investigate the consequences of the assumption $\bar{\lambda}>0$.

Lemma 2.2 Consider a solution $u \in C^{2}(\Omega) \cap C^{1}(\bar{\Omega})$ of (1.1) and assume that $f$ satisfies (H1) and (H2). Then the following properties hold

i) For all $\lambda>\bar{\lambda}$, we have $w_{\lambda}(x)>0$ for all $x \in \Sigma_{\lambda}$, 
If we further assume that $\bar{\lambda}>0$ then the following hold:

ii) For all $x \in \Sigma_{\bar{\lambda}}$ we have $w_{\bar{\lambda}}(x)>0$,

iii) If $x \in T_{\bar{\lambda}}$ and $\frac{\partial u}{\partial x_{1}}(x)=0$ then $\nabla u(x)=0$.

Proof: We start proving i). We first see that from Lemma 2.1 we have that $w_{\lambda}(x)>0$ for all $x \in \Sigma_{\lambda}$, for $\lambda<1$ but close to 1 . Next assume, for contradiction, that the lemma is false. Then for some $\lambda^{*}>\bar{\lambda}$ we have that $w_{\lambda}>0$ in $\Sigma_{\lambda}$ for all $\lambda>\lambda^{*}$ and there exist sequences $\left\{\lambda_{k}\right\}$ and $\left\{x_{k}\right\}$ such that $\left.\lambda_{k} \in\right] \bar{\lambda}, \lambda^{*}\left[\right.$ and $x_{k} \in \Sigma_{\lambda_{k}}$ with $\lim _{n \rightarrow+\infty} \lambda_{k}=\lambda^{*}$ and $w_{\lambda_{k}}\left(x_{k}\right) \leq 0$. Up to the extraction of a subsequence, we may assume that $\lim _{k \rightarrow+\infty} x_{k}=x^{*} \in \bar{\Sigma}_{\lambda^{*}}$ and $w_{\lambda^{*}}\left(x^{*}\right)=0$.

We see that $x^{*}$ cannot be in $\partial \Omega$ because $u>0$ in $\Omega$. If $x^{*} \in T_{\lambda^{*}}$ then, on one hand we have $\frac{\partial w_{\lambda^{*}}}{\partial x_{1}}\left(x^{*}\right)=-2 \frac{\partial u}{\partial x_{1}}\left(x^{*}\right)>0$ since $\lambda^{*}>\bar{\lambda}$. On the other hand, since $w_{\lambda_{k}}\left(x_{k}\right) \leq 0$ for any $k \in \mathbb{N}$, there exists $\theta_{k} \in(0,1)$ such that $\frac{\partial u}{\partial x_{1}}\left(\theta_{k} x_{k}+\left(1-\theta_{k}\right)\left(x_{k}\right)_{\lambda_{k}}\right) \geq 0$ and $\lim _{k \rightarrow+\infty}\left|\left(x_{k}\right)_{\lambda_{k}}-x_{k}\right|=0$. Thus $\frac{\partial u}{\partial x_{1}}\left(x^{*}\right) \geq 0$, obtaining a contradiction.

So we are left with the case $x^{*} \in \Sigma_{\lambda^{*}}$ and we will see that this is also impossible. We observe that since $\lambda^{*}>\bar{\lambda}$ then $\frac{\partial u}{\partial x_{1}}\left(x^{*}\right)<0$. As $w_{\lambda^{*}}(x) \geq 0$ for all $x \in \Sigma_{\lambda^{*}}$ we have that $\frac{\partial w_{\lambda^{*}}}{\partial x_{1}}\left(x^{*}\right)=0$ and $\frac{\partial u_{\lambda^{*}}}{\partial x_{1}}\left(x^{*}\right)>0$. Then, by the Implicit Function Theorem, there exists a neighborhood $\mathcal{V}$ of $\left(u\left(x^{*}\right), x_{2}^{*}\right) \in \mathbb{R}^{2}$ and two functions $v$ and $\bar{v}$ of class $C^{2}$ such that

$$
\begin{aligned}
t & =u\left(v\left(t, x_{2}\right), x_{2}\right) \quad \text { and } \\
t & =u_{\lambda^{*}}\left(\bar{v}\left(t, x_{2}\right), x_{2}\right) \quad \forall\left(t, x_{2}\right) \in \mathcal{V} .
\end{aligned}
$$

After some computations, we find that the function $v$ satisfies the quasilinear equation

$$
\left(1+\left(\frac{\partial v}{\partial x_{2}}\right)^{2}\right) \frac{\partial^{2} v}{\partial t^{2}}-2 \frac{\partial v}{\partial t} \frac{\partial v}{\partial x_{2}} \frac{\partial^{2} v}{\partial x_{2} \partial t}+\left(\frac{\partial v}{\partial t}\right)^{2} \frac{\partial^{2} v}{\partial x_{2}^{2}}=\left(\frac{\partial v}{\partial t}\right)^{3} f(t) \quad \text { in } \mathcal{V}
$$

A similar equation is satisfied by the function $\bar{v}$. It is easy to see that these equations are elliptic in $\mathcal{V}$. 
We may now consider the function $z\left(t, x_{2}\right)=v\left(t, x_{2}\right)-\bar{v}\left(t, x_{2}\right)$ that satisfies the equation

$$
\begin{gathered}
\left(1+\left(\frac{\partial v}{\partial x_{2}}\right)^{2}\right) \frac{\partial^{2} z}{\partial t^{2}}-2 \frac{\partial v}{\partial t} \frac{\partial v}{\partial x_{2}} \frac{\partial^{2} z}{\partial x_{2} \partial t}+\left(\frac{\partial v}{\partial t}\right)^{2} \frac{\partial^{2} z}{\partial x_{2}^{2}}+ \\
b_{1} \frac{\partial z}{\partial t}+b_{2} \frac{\partial z}{\partial x_{2}}=0 \quad \text { in } \mathcal{V},
\end{gathered}
$$

where the coefficients $b_{i}$ are given by

$$
\begin{aligned}
b_{1}\left(t, x_{2}\right)= & -2 \frac{\partial v}{\partial x_{2}} \frac{\partial^{2} \bar{v}}{\partial x_{2} \partial t}+\left(\frac{\partial v}{\partial t}+\frac{\partial \bar{v}}{\partial t}\right) \frac{\partial^{2} \bar{v}}{\partial x_{2}^{2}} \\
& +f(t)\left\{\left(\frac{\partial v}{\partial t}\right)^{2}+\frac{\partial v}{\partial t} \cdot \frac{\partial \bar{v}}{\partial t}+\left(\frac{\partial \bar{v}}{\partial t}\right)^{2}\right\}
\end{aligned}
$$

and

$$
b_{2}\left(t, x_{2}\right)=\frac{\partial^{2} \bar{v}}{\partial t^{2}}\left(\frac{\partial v}{\partial t}+\frac{\partial \bar{v}}{\partial t}\right)-\frac{\partial^{2} \bar{v}}{\partial x_{2} \partial t} \cdot \frac{\partial v}{\partial t} .
$$

We observe that all the coefficients of the differential operator are bounded. Since $z \leq 0$ in $\mathcal{V}$ and $z\left(u\left(x^{*}\right), x_{2}^{*}\right)=0$ we can use the Strong Maximum Principle to get that $z \equiv 0$ in $\mathcal{V}$. Thus near $x_{\lambda^{*}}^{*}$ we have $w_{\lambda^{*}} \equiv 0$. But this argument can be done at any point in $\Sigma_{\lambda^{*}}$, obtaining then that $w_{\lambda^{*}} \equiv 0$. This is impossible since $u>0$ in $\Omega$ and $w_{\lambda^{*}}(x)=u\left(x_{\lambda^{*}}\right)$ for all $x \in \partial \Omega \cap \bar{\Sigma}_{\lambda^{*}}$.

Thus, we have proved i), that is, for all $\lambda>\bar{\lambda}, w_{\lambda}>0$ in $\Sigma_{\lambda}$, so that passing to the limit we obtain $w_{\bar{\lambda}} \geq 0$. Now, if $w_{\bar{\lambda}}(\bar{x})=0$ for some $\bar{x} \in \Sigma_{\bar{\lambda}}$, we apply the Strong Maximum Principle as above to conclude that $w_{\bar{\lambda}} \equiv 0$, which is again impossible because $u>0$ in $\Omega$ as long as $\bar{\lambda}>0$. This proves assertion ii).

Now we prove iii). Let $\bar{x} \in T_{\bar{\lambda}}$ be such that $\frac{\partial u}{\partial x_{1}}(\bar{x})=0$ and $\nabla u(\bar{x}) \neq 0$. Then we have $\frac{\partial u}{\partial x_{2}}(\bar{x}) \neq 0$. We can then apply the same ideas as before, but now isolating $x_{2}$ instead of $x_{1}$. Since $w_{\bar{\lambda}}$ is strictly positive in $\Sigma_{\bar{\lambda}}$ we obtain by using the Hopf Lemma with the resulting differential operator that $-2 \frac{\partial u}{\partial x_{1}}(\bar{x})=\frac{\partial w}{\partial x_{1}}(\bar{x})>0$, a contradiction. This proves iii).

Our next lemma is crucial in our analysis. 
Lemma 2.3 Under the same hypothesis as in Lemma 2.2, assume $\bar{\lambda}>0$. Then for any $\bar{x} \in T_{\bar{\lambda}}$ such that $\frac{\partial u}{\partial x_{1}}(\bar{x})=0$ we have

$$
\frac{\partial^{2} u}{\partial x_{1}^{2}}(\bar{x})=0
$$

Proof: We first see that $\frac{\partial^{2} u}{\partial x_{1}^{2}}(\bar{x})>0$ implies that $\frac{\partial u}{\partial x_{1}}$ is positive at points to the right of $\bar{x}$, and this is not possible because of the definition of $\bar{\lambda}$.

Assume then, for contradiction, that $-\frac{\partial^{2} u}{\partial x_{1}^{2}}(\bar{x})=a>0$. We first observe that if $\lambda \in(0, \bar{\lambda})$ is close to $\bar{\lambda}$ then the function $x_{1} \rightarrow w_{\lambda}\left(x_{1}, \bar{x}_{2}\right)$ has a negative minimum in the interval $(\lambda, b)$, where $b>\bar{\lambda}$ is such that $\left(b, \bar{x}_{2}\right) \in \partial \Omega$. In fact, a Taylor development around $\bar{x}=\left(\bar{\lambda}, \bar{x}_{2}\right)$ gives

$$
\frac{\partial w_{\lambda}}{\partial x_{1}}\left(x_{1}, \bar{x}_{2}\right)=-2 a\left(x_{1}-\lambda\right)+o\left(\left|x_{1}-\bar{\lambda}\right|+\left|\left(2 \lambda-x_{1}\right)-\bar{\lambda}\right|\right),
$$

so that if $\bar{\lambda}-\lambda>0$ and $x_{1}-\lambda>0$ are small enough then $\frac{\partial w_{\lambda}}{\partial x_{1}}\left(x_{1}, \bar{x}_{2}\right)<0$.

Let $x_{1}(\lambda) \in(\lambda, b)$ be a point where the global minimum of the function $w_{\lambda}\left(x_{1}, \bar{x}_{2}\right)$ is achieved, then $w_{\lambda}\left(x_{1}(\lambda), \bar{x}_{2}\right)<0$ and

$$
\frac{\partial w_{\lambda}}{\partial x_{1}}\left(x_{1}(\lambda), \bar{x}_{2}\right)=0 .
$$

Next we claim that

$$
\lim _{\lambda \rightarrow \bar{\lambda}_{-}} \frac{\bar{\lambda}-\lambda}{x_{1}(\lambda)-\lambda}=0
$$

Here and in what follows $\lambda<\bar{\lambda}$, and $\lambda \rightarrow \bar{\lambda}_{-}$means $\lambda \rightarrow \bar{\lambda}$ and $\lambda<\bar{\lambda}$. In order to prove this claim we first recall that from Lemma $2.2 w_{\bar{\lambda}}(x)>0$ in $\Sigma_{\bar{\lambda}}$. Then, since $\lim _{\lambda \rightarrow \bar{\lambda}_{-}} w_{\lambda}(x)=w_{\bar{\lambda}}(x)$ for any $x \in \Sigma_{\bar{\lambda}}$, we have

$$
\lim _{\lambda \rightarrow \bar{\lambda}_{-}} \max \left\{x_{1}-\lambda \mid \lambda<x_{1} \text { and } w_{\lambda}\left(x_{1}, \bar{x}_{2}\right) \leq 0\right\}=0
$$

and consequently

$$
\lim _{\lambda \rightarrow \bar{\lambda}_{-}}\left(x_{1}(\lambda)-\lambda\right)=0
$$


Taking this into account we next consider a Taylor development of condition (2.4) which reads

$$
\begin{aligned}
0= & \frac{\partial u}{\partial x_{1}}\left(2 \lambda-x_{1}(\lambda), \bar{x}_{2}\right)+\frac{\partial u}{\partial x_{1}}\left(x_{1}(\lambda), \bar{x}_{2}\right) \\
= & \frac{\partial^{2} u}{\partial x_{1}^{2}}(\bar{x})\left(2 \lambda-x_{1}(\lambda)-\bar{\lambda}\right)+\frac{\partial^{2} u}{\partial x_{1}^{2}}(\bar{x})\left(x_{1}(\lambda)-\bar{\lambda}\right) \\
& +o\left(\left|2 \lambda-x_{1}(\lambda)-\bar{\lambda}\right|+\left|x_{1}(\lambda)-\bar{\lambda}\right|\right) \\
= & -2 \frac{\partial^{2} u}{\partial x_{1}^{2}}(\bar{x})(\bar{\lambda}-\lambda)+o\left(\left|2 \lambda-x_{1}(\lambda)-\bar{\lambda}\right|+\left|x_{1}(\lambda)-\bar{\lambda}\right|\right) .
\end{aligned}
$$

Assume now that the claim is not true, then up to a subsequence we would have $x_{1}(\lambda)-\lambda=O(\bar{\lambda}-\lambda)$ and then

$$
0=-2 \frac{\partial^{2} u}{\partial x_{1}^{2}}(\bar{x})(\bar{\lambda}-\lambda)+o(\bar{\lambda}-\lambda)
$$

which means that $\frac{\partial^{2} u}{\partial x_{1}^{2}}(\bar{x})=0$, a contradiction that proves the claim.

Now we give a rescaling argument. In the following arguments we keep $x_{2}=\bar{x}_{2}$ fixed, so that for notational convenience we will not be write it any more.

For $\lambda<\bar{\lambda}$ we define $\varepsilon(\lambda)=x_{1}(\lambda)-\lambda, \eta(\lambda)=u\left(\bar{x}_{1}\right)-u\left(x_{1}(\lambda)\right)$ and $\zeta(\lambda)=u_{\lambda}\left(\bar{x}_{1}\right)-u_{\lambda}\left(x_{1}(\lambda)\right)=u\left(\bar{x}_{1}\right)-u\left(2 \lambda-x_{1}(\lambda)\right)$, and we consider the rescaled functions $v^{\lambda}$ and $\bar{v}^{\lambda}$ defined in the interval $[0,1]$ as follows

$$
\begin{aligned}
& v^{\lambda}(y)=\eta^{-1}(\lambda)[u(\varepsilon(\lambda) y+\lambda)-u(\bar{x})] \quad \text { and } \\
& \bar{v}^{\lambda}(y)=\zeta^{-1}(\lambda)\left[u_{\lambda}(\varepsilon(\lambda) y+\lambda)-u(\bar{x})\right] \quad \text { for } \quad y \in[0,1] .
\end{aligned}
$$

Then we have

$$
-1=v^{\lambda}(1)=\min _{s \in(0,1)} v^{\lambda}(s) \leq v^{\lambda}(y) \leq \max _{s \in(0,1)} v^{\lambda}(s)=v^{\lambda}\left(\frac{\bar{\lambda}-\lambda}{\varepsilon(\lambda)}\right)=0,
$$

for all $y \in[0,1]$, and defining $\bar{v}^{\lambda}$ for argument less than 0 as in (2.6),

$$
-1=\bar{v}^{\lambda}(1)=\min _{s \in\left[-\frac{\bar{\lambda}-\lambda}{\varepsilon(\lambda)}, 1\right]} \bar{v}^{\lambda}(s) \leq \bar{v}^{\lambda}(y) \leq \max _{s \in\left[-\frac{\bar{\lambda}-\lambda}{\varepsilon(\lambda)}, 1\right]} \bar{v}^{\lambda}(s)=\bar{v}^{\lambda}\left(-\frac{\bar{\lambda}-\lambda}{\varepsilon(\lambda)}\right)=0,
$$


for any $y \in\left[-\frac{\bar{\lambda}-\lambda}{\varepsilon(\lambda)}, 1\right]$. By the change of variables we find that the functions $v^{\lambda}$ and $\bar{v}^{\lambda}$ satisfy the equations

$$
\begin{aligned}
-\frac{\partial^{2} v^{\lambda}}{\partial y^{2}} & =\frac{\varepsilon^{2}}{\eta}(\lambda) \cdot g^{\lambda}(y) \quad \text { and } \\
-\frac{\partial^{2} \bar{v}^{\lambda}}{\partial y^{2}} & =\frac{\varepsilon^{2}}{\zeta}(\lambda) \cdot h^{\lambda}(y),
\end{aligned}
$$

where $g^{\lambda}(y)=-\frac{\partial^{2} u}{\partial x_{1}^{2}}(\varepsilon(\lambda) y+\lambda)$ and $h^{\lambda}(y)=-\frac{\partial^{2} u}{\partial x_{1}^{2}}(\lambda-\varepsilon(\lambda) y)$. Since $g^{\lambda}$ and $h^{\lambda}$ converge uniformly to $-\frac{\partial^{2} u}{\partial x_{1}^{2}}(\bar{x})=a>0$, and since $\lim _{\lambda \rightarrow \bar{\lambda}_{-}} v^{\lambda}(0)=\lim _{\lambda \rightarrow \bar{\lambda}_{-}} \bar{v}^{\lambda}(0)=$ 0 and $\lim _{\lambda \rightarrow \bar{\lambda}_{-}} v^{\lambda}(1)=\lim _{\lambda \rightarrow \bar{\lambda}_{-}} \bar{v}^{\lambda}(1)=-1$, we find that

$$
\lim _{\lambda \rightarrow \bar{\lambda}_{-}} \frac{\varepsilon^{2}(\lambda)}{\eta(\lambda)}=\lim _{\lambda \rightarrow \bar{\lambda}_{-}} \frac{\varepsilon^{2}(\lambda)}{\zeta(\lambda)}=\frac{2}{a}, \text { and } \lim _{\lambda \rightarrow \bar{\lambda}_{-}} \frac{\partial v^{\lambda}}{\partial y}(1)=\lim _{\lambda \rightarrow \bar{\lambda}_{-}} \frac{\partial \bar{v}^{\lambda}}{\partial y}(1)=-2
$$

Then

$$
\frac{\partial u}{\partial x_{1}}\left(x_{1}(\lambda)\right)=\frac{\eta(\lambda)}{\varepsilon(\lambda)} \frac{\partial v^{\lambda}}{\partial y}(1)=-2 \frac{\eta(\lambda)}{\varepsilon(\lambda)}(1+o(1))
$$

and

$$
\frac{\partial u_{\lambda}}{\partial x_{1}}\left(x_{1}(\lambda)\right)=\frac{\zeta(\lambda)}{\varepsilon(\lambda)} \frac{\partial \bar{v}^{\lambda}}{\partial y}(1)=-2 \frac{\zeta(\lambda)}{\varepsilon(\lambda)}(1+o(1)),
$$

where $o(1) \rightarrow 0$ as $\lambda \rightarrow \bar{\lambda}_{-}$. From here and from the definition of $x_{1}(\lambda)$ we have

$$
0=\frac{\partial w_{\lambda}}{\partial x_{1}}\left(x_{1}(\lambda)\right)=\frac{\partial u_{\lambda}}{\partial x_{1}}\left(x_{1}(\lambda)\right)-\frac{\partial u}{\partial x_{1}}\left(x_{1}(\lambda)\right)=-2 \frac{\zeta(\lambda)-\eta(\lambda)}{\varepsilon(\lambda)}(1+o(1)) .
$$

But on the other hand we have

$$
\begin{aligned}
w_{\lambda}\left(x_{1}(\lambda)\right) & =u_{\lambda}\left(x_{1}(\lambda)\right)-u\left(x_{1}(\lambda)\right) \\
& =\left[u_{\lambda}\left(x_{1}(\lambda)\right)-u(\bar{x})\right]-\left[u\left(x_{1}(\lambda)\right)-u(\bar{x})\right] \\
& =\zeta(\lambda)-\eta(\lambda)<0,
\end{aligned}
$$

providing a contradiction. $\square$ 


\section{Proof of the main theorems.}

In this section we give the proof of Theorems 1.1 and 1.2. The idea is to use the moving planes method to reach a critical position in a given direction and apply Lemma 2.3. Then to perturb slightly the direction to prove that $\Delta u=0$ at a critical point and apply hypothesis (H3). Here is where we use the 2-dimensionality assumption. Instead of proving Theorems 1.1 and 1.2. we will prove some more general versions where we consider a larger class of domains.

In the first place we will state our more general version of Theorem 1.1

Theorem 3.1 Let $\Omega \subset \mathbb{R}^{2}$ be a bounded open domain and $\gamma \in S^{1}$. We assume that $\Omega$ is $\gamma$-convex, symmetric with respect to a line $T$ orthogonal to $\gamma$, and satisfies (O1)-(O2). We assume that $f$ satisfies (H1), (H2) and (H3). If $u \in C^{2}(\Omega) \cap C^{1}(\bar{\Omega})$ is a solution of (1.1) then $u$ is symmetric with respect to $T$ and $\nabla u(x) \cdot \gamma<0$ for any $\gamma \in S^{1}$ and $x \in \Omega$ such that

$$
x \cdot \gamma>\sup \{y \cdot \gamma \mid y \in \partial \Omega, \quad \nu(y) \cdot \gamma=0\},
$$

where $\nu(y)$ is the outgoing normal at $y \in \partial \Omega$.

Clearly Theorem 1.1 is a particular case of Theorem 3.1.

Proof of Theorem 3.1. We will keep the notational conventions given in Section 2. In particular we recall that $\bar{\lambda}=\inf \left\{\lambda \in(0,1) \mid \frac{\partial u}{\partial x_{1}}(\bar{x})<0\right.$ in $\left.\Sigma_{\lambda}\right\}$. By Lemma 2.1 we have that $\bar{\lambda}$ belongs to $[0,1)$.

Our main goal is to prove that $\bar{\lambda}=0$. If this was not the case then there would exist an $\bar{x} \in T_{\bar{\lambda}}$ such that $\nabla u(\bar{x})=0$ and $\frac{\partial^{2} u}{\partial x_{1}^{2}}(\bar{x})=0$ as we obtain from Lemmas 2.2 and 2.3.

Next we claim that $\frac{\partial^{2} u}{\partial x_{2}^{2}}(\bar{x})=0$ also. First choose, among those $\bar{x} \in T_{\bar{\lambda}}$ such that $\frac{\partial u}{\partial x_{1}}(\bar{x})=0$, the one with the largest $x_{2}$-component. This point, we keep calling $\bar{x}$ does not belong to $\partial \Omega$ by Lemma 2.1. Now we slightly rotate the direction $e_{1}$ in a positive angle $\theta$ to obtain $\gamma$. And we perform the 
moving planes argument already given but now in the direction $\gamma$. Here we have to use a slightly different version of Lemma 2.1:

For any $\eta>0$, there exists $\varepsilon>0$ such that if $x \in \bar{\Omega}$ is such that $x \cdot \gamma \geq$ $\sup \{y \cdot \gamma \mid y \in \partial \Omega$ and $\nu(y) \cdot \gamma=0\}+\eta$ and $\operatorname{dist}(x, \partial \Omega) \leq \varepsilon$ then $\nabla u(x) \cdot \gamma<0$.

The moving plane will stop because some point $x$ in $T_{\lambda}(\gamma) \cap \Omega$ satisfies $\nabla u(x)=0$. We call $x(\theta)$ the point with vanishing gradient with largest vertical component. Here we have used hypothesis (O2) to assure that the moving plane was stopped because the gradient of $u$ vanishes and not because of a geometrical obstruction.

Now we have two posibilities. First, if $x(\theta)=\bar{x}$ then the application of Lemma 2.3 will imply that the second derivative of $u$ in the direction $\gamma$ vanishes, and thus proving the claim. Second, assume that $x(\theta) \neq \bar{x}$ for all small $\theta$. In this case we first observe that $x(\theta) \rightarrow \bar{x}$ because of our choice of $\bar{x}$, and then using that $\nabla u$ vanishes both at $\bar{x}$ and $x(\theta)$ and the Mean Value Theorem we find that for all small $\theta$ the second derivative of $u$ in the direction $\tau(\theta)=(x(\theta)-\bar{x}) /|x(\theta)-\bar{x}|$ vanishes at some intermediate point. Then, taking limit as $\theta \rightarrow 0_{+}$, and noting that $\tau(\theta)$ converges to $e_{2}$ we prove the claim.

As a conclusion we have now that $\Delta u(\bar{x})=0$ and then $f(u(\bar{x}))=0$. By Lemma 2.2 we have that $w_{\bar{\lambda}}(x)=u_{\bar{\lambda}}(x)-u(x)>0$ for all $x \in \Sigma_{\bar{\lambda}}$, and of course $w_{\bar{\lambda}}$ satisfies the equation

$$
\Delta w_{\bar{\lambda}}+\left\{\frac{f\left(u_{\bar{\lambda}}(x)\right)-f(u(x))}{u_{\bar{\lambda}}(x)-u(x)}\right\} w_{\bar{\lambda}}=0 \quad x \in \Sigma_{\bar{\lambda}} .
$$

Using hypothesis (H3) we then obtain that

$$
\Delta w_{\bar{\lambda}}+C w_{\bar{\lambda}} \leq 0 \quad x \in \Sigma_{\bar{\lambda}}
$$

so that $\frac{\partial w_{\bar{\lambda}}}{\partial x_{1}}(\bar{x})>0$. But $\frac{\partial w_{\bar{\lambda}}}{\partial x_{1}}(\bar{x})=-2 \frac{\partial u(\bar{x})}{\partial x_{1}}(\bar{x})>0$ which is impossible. This contradiction proves that $\bar{\lambda}=0$.

To complete the proof we proceed in standard way. First we conclude that $u\left(-x_{1}, x_{2}\right) \geq u\left(x_{1}, x_{2}\right)$ by Lemma 2.2. Next we repeat the argument from the other side, and obtain then that $u\left(-x_{1}, x_{2}\right)=u\left(x_{1}, x_{2}\right)$. 
The conclusion on the derivative is implicit in the moving planes scheme.

In what follows we consider the extension of Theorem 3.1 to the case of positive solutions of semilinear elliptic equations in unbounded domains.

Let us start describing the adequate assumptions we need to consider on the unbounded domain $\Omega$ for our results to be true. Given $\gamma \in S^{1}$, we say that $\Omega$ is $\gamma$-conic if for any $x \in \Omega$ the set $\{t \in \mathbb{R} \mid x-t \gamma \in \Omega\}$ is a semiinfinite interval bounded from below. We note that in this case $\tilde{\Sigma}_{\lambda}(\gamma) \subset \Omega$ for all $\lambda \in \mathbb{R}$. Our hypothesis is the following

(O3) $\Omega$ is $\gamma$-conic and for all $\lambda \in \mathbb{R}$ the cap $\Sigma_{\lambda}(\gamma)$ is bounded. Moreover for every $\lambda_{s} \in \mathbb{R}$ and for every $\varepsilon>0$ there exists $\delta>0$ so that for any $d \in S^{1},|d-\gamma|<\delta$ we have $\Sigma_{\lambda}(d) \subset \Omega$ for all $\lambda>\lambda_{s}+\varepsilon$.

We observe that the set of directions satisfying (O3) is open in $S^{1}$.

Then our general theorem is the following

Theorem 3.2 Assume that $\Omega \subset \mathbb{R}^{2}$ is an open domain and $\gamma \in S^{1}$. Assume that $\Omega$ is $\gamma$-conic and assume that $\Omega$ satisfies (O1), (O3) and that $f$ satisfies (H1), (H2) and (H3). If $u \in C^{2}(\Omega) \cap C^{1}(\bar{\Omega})$ is a solution of (1.1) then $u$ is strictly decreasing in the direction $\gamma$, actually $\nabla u(x) \cdot \gamma<0$ for any $x \in \Omega$.

We see that Theorem 1.2 is a direct consequence of Theorem 3.2.

Proof of Theorem 3.2. The proof of Theorem 3.2 follows the steps of the proof above. Working with the direction $\gamma=e_{1}$ for simplicity, we let $\lambda_{*}=\sup \left\{\lambda \mid \Sigma_{\lambda} \neq \emptyset\right\}$ and we define $\bar{\lambda}=\inf \left\{\lambda \in\left(-\infty, \lambda_{*}\right) \mid \frac{\partial u}{\partial x_{1}}(\bar{x})<0\right.$ in $\left.\Sigma_{\lambda}\right\}$

Making the obvious changes, we easily see that Lemma 2.1 with arbitrary $\eta \in\left(-\infty, \lambda_{*}\right)$, and Lemma 2.2 and 2.3 remain valid in this new context. In particular we can start the moving planes argument. Then we show that $\bar{\lambda}$ cannot be finite because the contrary leads to a contradiction as in the proof of Theorem 3.1. Here we use the hypothesis (O3).

Thus $\bar{\lambda}=-\infty$, and then the conclusion of the theorem follows. 


\section{References}

[1] A. D. Alexandroff, "A characteristic property of the spheres", Ann. Mat. Pura Appl. Vol. 58, 303-354, 1962.

[2] H. Berestycki \& L. Nirenberg, "On the method of moving planes and the sliding method", Bol. Soc. Bras. Mat. Vol. 22 (1), 1-37, 1991.

[3] H. Berestycki, L. Caffarelli \& L. Nirenberg, "Monotonicity for elliptic equations in unbounded Lipschitz domains", Comm. Pure Appl. Math. Vol. 50 (11), 1089-1111, 1997.

[4] F. Brock, "Continuous Steiner Symmetrization", Math. Nachrichten Vol. 172, 25-48, 1995.

[5] F. Brock, "Continuous Rearrangement and Symmetry of Solutions of Elliptic Problems", Preprint.

[6] C. Cortázar, M. Elgueta \& P. Felmer, "On a semilinear elliptic problem in $\mathbb{R}^{N}$ with a non-Lipschitzian non-linearity", Advances in Differential Equations Vol. 1 (2), 199-218, 1996.

[7] C. Cortázar, M. Elgueta \& P. Felmer, "Symmetry in an elliptic problem and the blow-up set of a quasilinear heat equation", Comm. in Partial Diff. Equations, Vol. 21 (3\&4), 507-520, 1996.

[8] J. Dolbeault \& P. Felmer, "Monotonicity up to radially symmetric cores of positive solutions to nonlinear elliptic equations: local moving planes and unique continuation in a non-Lipschitz case", Preprint.

[9] M. J. Esteban \& P. L. Lions, "Existence and nonexistence results for semilinear elliptic problems in unbounded domains", Proc. Roy. Soc. Edinburgh Sect. A Vol. 93, (1-2), 1-14, 1982/83. 
[10] B. Gidas, W. M. Ni \& L. Nirenberg, "Symmetry and Related Properties via the Maximum Principle", Commun. Math. Phys. Vol. 68, 209-243, 1979 .

[11] B. Gidas, W. M. Ni \& L. Nirenberg, "Symmetry of positive solutions of nonlinear elliptic equations in $\mathbb{R}^{N}$, Advances in Math. Studies 7 A, 209-243, 1979.

[12] C. Gui, "Symmetry of the blow up set of a porous medium type equation", Comm. Pure Appl. Math. Vol. XLVIII, 471-500, 1995.

[13] H. G. Kaper, M. K. Kwong \& Y. Li, "Symmetry Results for Reaction Diffusion Equations", Differential Integral Equations Vol. 6 (5), 10451056, 1993.

[14] C. Li, "Monotonicity and symmetry of solutions of fully nonlinear elliptic equations in unbounded domains", Comm. in Partial Diff. Equations Vol. 16 (4\&5), 585-615, 1991.

[15] Y. Li \& W.-M. Ni, "Radial symmetry of positive solutions of nonlinear elliptic equations in $R^{N}$, Comm. in Partial Diff. Equations Vol. 18 (5\&6), 1043-1054, 1993.

[16] P.-L. Lions, "Two geometrical properties of solutions of semilinear problems", Applicable Anal. Vol. 12 (4), 267-272, 1981.

[17] L. A. Peletier \& J. Serrin, "Uniqueness of nonnegative solutions of semilinear equations in $R^{N "}$, J. Diff. Eq. Vol. 61, 380-397, 1986.

[18] J. Serrin, "A symmetry problem in potential theory", Arch. Rat. Mech. Anal. Vol. 43, 304-318, 1971. 